\title{
Association of Dietary Inflammatory Index (DII) With the Risk of Poly Cystic Ovary Syndrome: a Case- control Study
}

\section{Parivash Kavei}

Lorestan University of Medical Sciences

Esmaeil Yousefi Rad

Lorestan University of Medical Sciences

Soheila Akbari

Lorestan University of Medical Sciences

Ebrahim Falahi

Lorestan University of Medical Sciences

Mahnaz Mardani

Lorestan University of Medical Sciences

Mehdi Birjandi

Lorestan University of Medical Sciences

Somayeh Saboori ( $\nabla$ saburi_somaye@yahoo.com )

Nutritional Health Research Center, Department of Nutrition, Lorestan University of Medical Sciences

https://orcid.org/0000-0001-5435-2878

\section{Research note}

Keywords: PCOS, inflammation, dietary inflammatory index (DII), case-control

Posted Date: June 25th, 2021

DOI: https://doi.org/10.21203/rs.3.rs-645990/v1

License: (1) (1) This work is licensed under a Creative Commons Attribution 4.0 International License. Read Full License 


\section{Abstract}

Objective: The aim of this case-control study was to investigate the association between Dietary Inflammatory Index (DII) and PCOS risk in the 120 newly-diagnosed cases of PCOS and 120 healthy controls aged between 18-45 years in Khorramabad of Iran.

Results: Energy intake was significantly more in PCOS patients $(p=0.01)$ and these people were less active than control women $(p=0.001)$. The mean \pm SD of DII in PCOS patients was $0.4 \pm 2.09$ while it was $0.45 \pm 1.92$ in control group ( $p=0.001)$. There was a positive association between increasing DII score and the risk of PCOS with the OR of $2.41(1.15,5.02)$ in the crude model when we compared the fourth quartile with the lowest one. This association was still significant in several models after adjusting for age and energy intake in the model 1 (P-value for trend=0.001), in the second model and after adjusting for the level of activity, education, and family history of PCOS along with the first model (P-value for trend 0.003 ), and finally after additional adjustment for BMI in model 3 (P-value for trend 0.003). Results of present study showed that consuming more pro-inflammatory diets with higher DII scores are associated with increased risk of PCOS.

\section{Introduction}

Polycystic ovary syndrome (PCOS) is the most common endocrine metabolic disorder in women of childbearing ages. Various factors are involved in its occurrence, the disease may exist as a genetic predisposition in a person that is influenced by environmental factors such as eating habits, lifestyle and social status [1-5]. The prevalence of this syndrome in the worldwide is estimated in the range of 4 to $12 \%$ [6]. In Iran, several studies on the prevalence of PCOS have been conducted with different results dependent to the criteria were used. This prevalence was estimated in one community based study in different regions of Iran (Qazvin (center), Kermanshah (west), Golestan (north) and Hormozgan (south)), from 7.1\% according to the $\mathrm{NIH}$ criteria, $14.6 \%$ according to the Rotterdam criteria and to $11.7 \%$ according to the AES criteria [7]. One systematic review study was estimated the prevalence of PCOS up to $8.6 \%, 19.5 \%$ and $41 \%$ according to the NIH criteria, Rotterdam criteria and based on the ultrasound method, respectively [8]. Complications of PCOS include increases the risk of reproductive problems such as infertility, decreased ovulation, irregular menstrual cycles, ovarian disorders, high levels of male hormones such as testosterone that can cause unwanted hair growth and acne, endometrial cancer and late menopause. It can also affect the quality of life with depression, low self-esteem, and anxiety, as well as a number of metabolic disorders including diabetes with high insulin levels or insulin resistance, and cardiovascular disease associated with high blood pressure and lipid disorders such as abnormal cholesterol level [9-16].

PCOS is a pro-inflammatory condition, and studies showed that chronic low-grade inflammation predisposes to metabolic abnormalities and ovarian dysfunction and there is a strong association between hyper-androgenism and inflammation in PCOS subjects [17]. Excess glucose levels observed in PCOS patients induces an inflammatory response by increasing ROS-induced oxidative stress. Thus, diet-induced inflammation in PCOS leads to pro-inflammatory signaling, which is involved in the development of insulin resistance [18]. PCOS patients had a genetic predisposition for low-grade chronic inflammation and several 
pro-inflammatory genotypes, including those encoding TNFa and the TNF receptor type 2, as well as interleukin-6 (IL-6) and its signal transducer, are associated with this syndrome[19].

DII (Dietary Inflammatory Index) is a scoring algorithm based on an extensive review of articles published between 1950 and 2010 by Shivapa et al. This index evaluates the role of various dietary parameters on the levels of inflammatory factors such as IL-1 $\beta$ IL-4区 IL-6区 IL-10区 TNF- $\alpha$ and CRP. Shivapa and colleagues studied the inflammatory potential of 45 food items. Scores of $+1,-1$ and zero, respectively are considered as pro-inflammatory, anti-inflammatory and neutral foods, which respectively increase, decrease and have no effect on the above inflammatory factors. this index has been expanding since 2009 and was updated in $2014[20]$. The purpose of creating it was to provide a tool to evaluate the inflammatory potential of the diet based on the pro-inflammatory and anti-inflammatory properties of various dietary components including macronutrients, vitamins, minerals, flavonoids and specific nutrients according to the previous studies on cell culture as well as animal and human studies [21, 22]. A high score of DII indicates a pro-inflammatory diet and a low score indicates an anti-inflammatory diet. This index, whose validity and reliability have recently been evaluated by two dietary evaluation methods, was able to predict hs-CRP values greater than 3 $\mathrm{mg} / \mathrm{L}[23]$.

According to the previous studies, a diet with high DII is strongly associated with metabolic syndrome, hypertension, elevated triglycerides, decreased HDL, and cardiovascular disorders, as well as increased inflammatory factors. In recent years, several studies have been performed in western countries which was assessing the relationship between western dietary patterns and DII with various diseases [21, 24-26]. To the best of our knowledge, no study has been done on the relationship between DII and PCOS. Therefore, the present study aimed to investigate the association between dietary inflammatory index and PCOS in Iranian women.

\section{Methods}

The present study was a case-control designed study on 120 newly-diagnosed cases of PCOS and 120 healthy controls aged between 18-45 years in Khorramabad of Iran in 2019-2020. Participants were selected using consecutive random sampling method. All participants signed a written informed consent before data gathering. Rotterdam criteria was used for diagnosis of PCOS. The inclusion criteria in the current study were as age ranges of 18-45 years, no history of chronic diseases including diabetes, liver, thyroid, cardiovascular and kidney diseases, not following a special diet, no use of appetite suppressants or anti-obesity drugs, having PCOS with no more than 6 months after its diagnosis and no use of insulin and metformin. People with eating disorders, use from diet or exercise for weight loss, smoking, alcohol and use of any multivitamin and mineral supplements were excluded from the study. In order to increase the comparability between the study groups, we matched the case and control groups in terms of age (18-30, 31-35 and 36-45 year) and marital status and the effects of other confounding variables including BMI, education, physical activity, marital status and family history of PCOS were adjusted by including them into the different statistical models. This study was approved by the ethics committee of Lorestan University of Medical Sciences with the ethics code of: IR.LUMS.REC.1398.058. 
A validated 168-item Food Frequency Questionnaire (FFQ) was used to assess dietary intakes of the study participants. FFQ presents a list of food items and a standard serving size for each one. Participants should report the frequency of their food consumption during the previous year (frequency of food items on daily, weekly, or monthly intake [27]. Also, in order to calculate the dietary inflammatory index, it was necessary to have information about the intake of some foods such as spices including saffron, ginger, turmeric, black pepper, rosemary and thyme, which are not available in FFQ. As a result, some additional questions regarding the intake of these foods were asked of the interviewees. The questionnaire was completed by face-to-face interview. During the interview, the average size of each food item in the food frequency questionnaire was explained to the case and control groups. After completing the food frequency questionnaire for all individuals in the case and control groups, Software program Nutritionist IV was used for nutrient analysis which was modified for Iranian foods and the daily intake of each person in terms of total energy, protein, carbohydrates, fiber, total fat, fatty acids PUFA, MUFA, SFA and food groups were determined.

The DII was calculated based on the intakes of 45 food parameters obtained from the FFQ whose inflammatory score, mean and SD of the global intake of each nutritional parameter were calculated [20]. To calculate the DII score, energy-adjusted values of these items were calculated, firstly. Then, the values obtained for each variable were subtracted from the corresponding mean global intake and divided by the global SD to obtain the $z$ score. The z-score obtained was then converted to a centered percentile score in order to reduce skewness and this percentage score for each food parameter was multiplied by 2 and subtracting 1 , So that its range is between -1 to +1 . In the next step, the numbers obtained for each of the food parameters were multiplied by the corresponding inflammatory score and then the inflammation score of all of the food parameters were summed to obtain the total inflammatory score for each person. A higher DIl score (more positive) indicates a pro-inflammatory diet and a lower score (more negative) indicates an anti-inflammatory diet. The minimum of the DII score is -8.87 , while the maximum score is +7.98 [20].

In this study, from a total of 45 nutrients, 36 dietary parameters were used to calculate the DII including energy, carbohydrates, protein, total fat, monounsaturated fatty acids (MUFA), polyunsaturated fatty acids (PUFA), saturated fatty acids (SFA), cholesterol, omega 3 , omega 6 , fiber, thiamine, riboflavin, niacin, vitamin B6, folic acid, vitamin B12, vitamin A, vitamin C, vitamin E, vitamin D, beta-carotene, iron, Selenium, zinc, magnesium, caffeine, tea, onion, garlic, turmeric, saffron, pepper, ginger, thyme and rosemary.

Anthropometric parameters of participating women, including weight, height, waist circumference, hip circumference and BMI were measured. Measurements were performed according to standard protocol. Height, without wearing shoes in a standing position, using a measuring tape was measured and recorded with an accuracy of $0.1 \mathrm{~cm}$. Weight was measured while the subjects were light clothed and without wearing shoes, using a Seka scale (made in Germany) with an accuracy of $100 \mathrm{~g}$. Waist circumference (WC) was measured using a flexible anthropometric tape in the midway between the lowest ribs margin and the iliac crest at the level of the umbilicus with the least possible coverage, with an accuracy of $0.1 \mathrm{~cm}$ and the hip circumference was measured using a flexible anthropometric tape in the widest part of the pelvis with the least possible coverage. Also, body mass index (BMI) was calculated by dividing weight by height squared ( $\mathrm{kg} / \mathrm{m} 2)$. 
A general questionnaire was used to collect additional variables such as socio-economic status information including age, education, occupation, monthly income, marital status, and drug use (any types of drugs) and intake of nutritional supplements (minerals, vitamins). The data related to physical activity data were collected using the International Physical Activity Questionnaire (IPAQ) [28-31].

The Kolmogorov-Smirnoff test was used to evaluate whether or not the distributions of the variables were normal. Mean values for cases and controls were compared using the Student's T-test and the means of more than two groups values were assessed using analysis of variance (ANOVA) for normally distributed variables. The chi-square test was used for comparing distribution of categorical variables. Logistic regression test was used to estimate odds ratios (ORs) and 95\% confidence intervals (Cls) which were adjusted for multiple covariates in different models. DII scores were analyzed as quartiles. Statistical tests were performed using SPSS software (SPSS 21). The $p$-values $<0.05$ were considered as statistically significant.

\section{Results}

Scores of DII in current study were between -4.66 (most anti-inflammatory score) to +5.6 (most proinflammatory score). The characteristics of 240 participants including 120 in case group women with PCOS and 120 in control group is shown in Table 1. Controls and cases were matched in age and marital status. The mean age of case participants was $31.9 \pm 6.9$ years and for women in control group was $32.9 \pm 6.1$ years. Significant differences were seen in physical activity level (1097.68 \pm 256 vs. $1503.8 \pm 484, p=0.001)$, Energy intake $(3220.1 \pm 856$ versus $2933.6 \pm 873, p=0.01)$ and DII scores $(0.4 \pm 2.09$ versus $-0.45 \pm 1.92 p=$ 0.001 ) between case and control groups, respectively. Cases were more likely to be positive in family history of PCOS than controls $(p=<0.001)$. Participant's characteristics among categories of DII is shown in Table 2. There were some differences across DII categories in energy intake and education level and participants in the fourth quartile of DII have more intake of energy. 
Table 1

Characteristics of study participants in a Case-Control Study of Iranian women with poly cystic ovary syndrome (PCOS)

\begin{tabular}{|c|c|c|c|}
\hline Characteristics & Case & Control & *P-value \\
\hline participants & 120 & 120 & \\
\hline Age, (years): mean \pm SD & $31.9 \pm 6.9$ & $32.9 \pm 6.1$ & 0.23 \\
\hline Energy intake (kcal); mean \pm SD & $3220.1 \pm 856$ & $2933.6 \pm 873$ & 0.01 \\
\hline $\mathrm{BMI}, \mathrm{kg} / \mathrm{m} 2$ mean $\pm \mathrm{SD}$ & $25.7 \pm 3.6$ & $25.3 \pm 4.1$ & 0.48 \\
\hline Dietary Inflammatory Index (DII): mean \pm SD & $0.4 \pm 2.09$ & $-0.45 \pm 1.92$ & 0.001 \\
\hline Physical activity (Met-Min/Week) & $1097.68 \pm 256$ & $1503.8 \pm 484$ & 0.001 \\
\hline \multicolumn{4}{|l|}{ Family history of PCOS (\%) } \\
\hline yes & $27(22.5 \%)$ & $2(1.7 \%)$ & \multirow[t]{2}{*}{$<0 / 001$} \\
\hline no & $93(77.5 \%)$ & 118(98.3) & \\
\hline \multicolumn{4}{|l|}{ Education, n (\%) } \\
\hline No formal education & $1(66.7 \%)$ & $2(33.3 \%)$ & \multirow[t]{4}{*}{0.26} \\
\hline Under Diploma & $18(45 \%)$ & $22(55 \%)$ & \\
\hline Diploma & $55(57.3 \%)$ & $41(42.7 \%)$ & \\
\hline Post Diploma & $45(44.6 \%)$ & $56(55.4 \%)$ & \\
\hline \multicolumn{4}{|l|}{ Marital status, (\%) } \\
\hline Married & $107(50 \%)$ & $107(50 \%)$ & \multirow[t]{2}{*}{1.00} \\
\hline Single & $13(50 \%)$ & $13(50 \%)$ & \\
\hline
\end{tabular}


Table 2

Participant characteristics by the level of dietary inflammatory index (DII), a Case-Control Study of Iranian women with poly cystic ovary syndrome (PCOS)

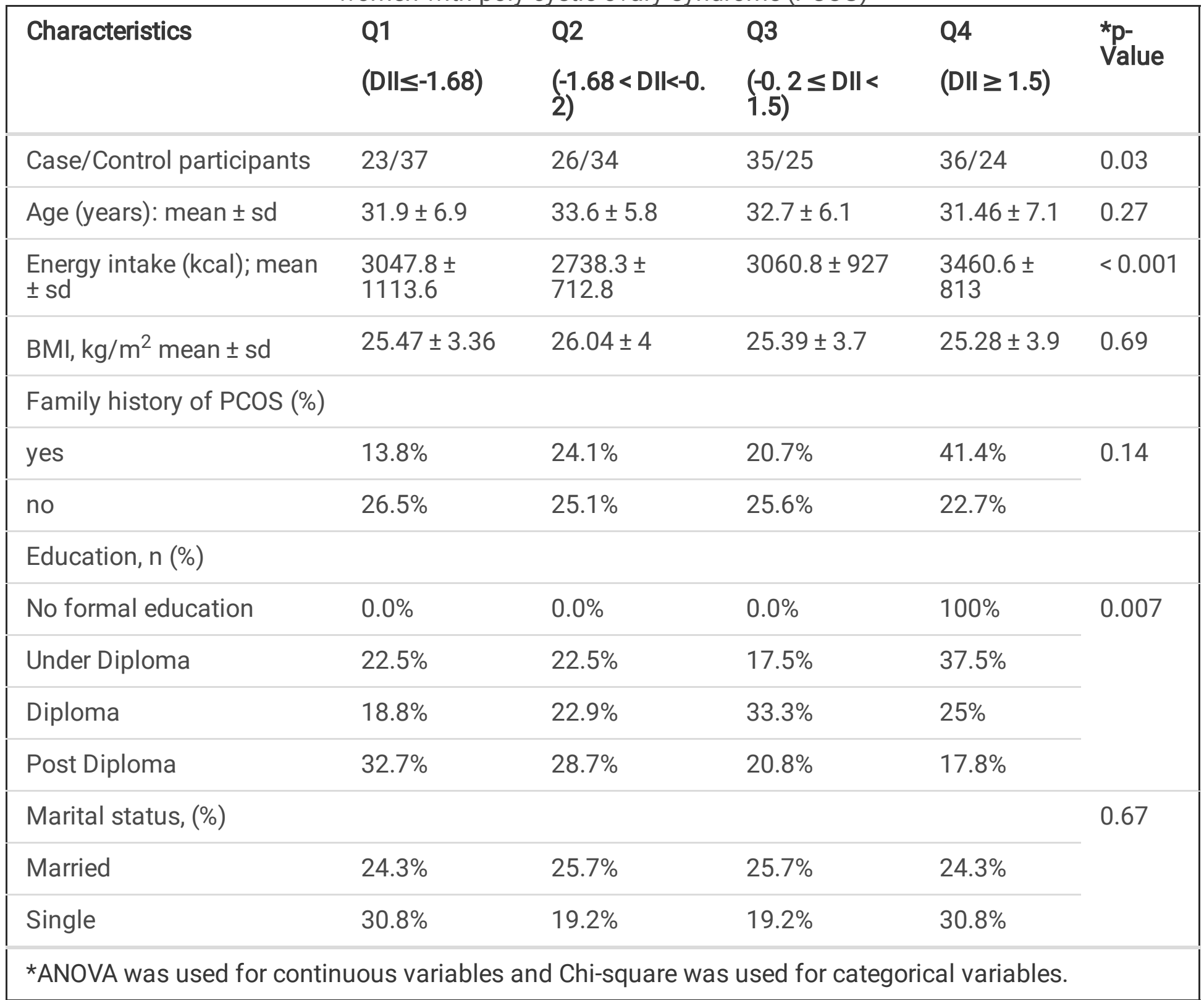

ORs and $95 \%$ Cls for the risk of PCOS are provided in Table 3. Results of crude model showed that there is a positive association between increasing DII score and the risk of PCOS with the OR of $1.23(95 \% \mathrm{Cl}=$ $1.08,1.41)$ when the energy adjusted DII (E-DII) score was fit as a continuous variable. The same results also seen when modeling the DII as quartiles. After adjusting age and energy intake in the model 1 , the OR for the highest quartile compared with the lowest quartile was $3.05(95 \% \mathrm{Cl}=1.4,6.63$; $\mathrm{P}$ - value for trend $=0.001)$. Again, in the second model and after adjusting for the level of activity, education, and family history of PCOS along with the first model, the results were similar to the model 1. (OR quartile 4 versus quartile $1=$ $2.79,95 \% \mathrm{Cl}=1.23,6.3 ; \mathrm{P}$ - value for trend 0.003 ). Additional adjustment for $\mathrm{BMI}$ in model 3 also showed the same results (OR quartile 4 versus quartile $1=2.81,95 \% \mathrm{Cl}=1.25,6.4$; $\mathrm{P}$ - value for trend 0.003 ). 
Table 3

Odds Ratios and 95\% Confidence Intervals for the association between DII and PCOS: A Case-Control Study of Iranian women

\begin{tabular}{|c|c|c|c|c|c|c|}
\hline & \multicolumn{4}{|c|}{ DII (quartiles) } & \multirow{2}{*}{$\begin{array}{l}\text { P- } \\
\text { value } \\
\text { for } \\
\text { trend }\end{array}$} & \multirow{2}{*}{$\begin{array}{l}\text { DII } \\
\text { (Continuous) }\end{array}$} \\
\hline & $\begin{array}{l}\text { Q1 } \\
\text { (DIl } \leq-1.68)\end{array}$ & $\begin{array}{l}\mathrm{Q} 2(-1.68< \\
\mathrm{D} \mid 1<-0.2)\end{array}$ & $\begin{array}{l}\text { Q3 }(-0.2 \leq \text { DII } \\
<1.5)\end{array}$ & $\mathrm{Q} 4$ (DII $\geq 1.5)$ & & \\
\hline & $\begin{array}{l}\mathrm{OR}(95 \% \\
\mathrm{Cl})\end{array}$ & OR $(95 \% \mathrm{Cl})$ & OR $(95 \% \mathrm{Cl})$ & OR $(95 \% \mathrm{Cl})$ & & OR $(95 \% \mathrm{Cl})$ \\
\hline $\begin{array}{l}\text { Crude } \\
\text { Model }\end{array}$ & 1.0 & $1.23(0.59,2.55)$ & $2.25(1.08,4.67)$ & $2.41(1.15,5.02)$ & 0.006 & $1.23(1.08,1.41)$ \\
\hline Model & 1.0 & $1.13(0.53,2.41)$ & $2.42(1.14,5.1)$ & $3.05(1.4,6.63)$ & 0.001 & $1.3(1.14,1.51)$ \\
\hline $\begin{array}{l}\text { Model } \\
\text { II }\end{array}$ & 1.0 & $1.02(0.47,2.1)$ & $2.16(0.99,4.7)$ & $2.79(1.23,6.3)$ & 0.003 & $1.27(1.09,1.47)$ \\
\hline $\begin{array}{l}\text { Model } \\
\text { III }\end{array}$ & 1.0 & $1.01(0.47,2.1)$ & $2.1(1.0,4.7)$ & $2.8(1.25,6.4)$ & 0.003 & $1.28(1.09,1.49)$ \\
\hline
\end{tabular}

\section{Discussion}

This case-control study was carried out for assessing the association between inflammatory diets which was measured by DII index and the risk of PCOS incidence. The results showed that in compared to control group, PCOS patients had more pro-inflammatory diets with increased DII scores. This result is consistent with the results of several studies assessing the relationship between DII and other inflammatory diseases. In the Kim HY, et al study it was shown that higher DII scores had positive association with the increased prevalence of hyperglycemia and central obesity respectively in men and post-menopausal women [32]. Another study on diabetic type 2 patients revealed that the odds ratio (OR) of disease was tripled in patients with highest quintile of DII in compared to the lowest one [33]. However, in another study on firefighters in Iran, although the CRP level was significantly more in the higher DII tertile, it was not associated with DII scores after adjusting confounding factors such as BMI and physical activity level [34].

Pro-inflammatory cytokines secreted from adipose tissue can induce insulin resistance by increasing the phosphorylation of serine/threonine residues in the docking protein insulin receptor substrate-1 (IRS-1) [35]. In other hand, inflammatory markers such as TNF-a can cause insulin resistance in visceral tissues through activating c-Jun $\mathrm{N}$-terminal kinase 1 and 2 (JNK1/2) [36]. Epidemiological studies have shown that insulin resistance could increase the risk of cardiovascular diseases $[37,38]$ and also it is related to increased incidence of some neoplasms including breast, colorectal and prostate cancers [39, 40]. PCOS is a low grade inflammatory disease and pro-inflammatory markers including interleukin 6 (IL-6) and tumor necrosis factor- $a$ (TNF- $a$ ) are increases in these patients which can induce hyperandrogenism seen in the PCOS patients $[41,42]$. 
One of the most common manifestations of PCOS is insulin resistance and resulting hyperinsulinemia which can affect nearly $50-70 \%$ of these patients. This situation can trigger hyperandrogenism and resulted in chronic oligo- or anovulation via elevating pro-inflammatory markers [43]. Human diet can influence inflammation via its diverse effects on pro-inflammatory and anti-inflammatory markers secretion. For instance, western diet which characterized by high levels of red meat and refined grains consumption has pro-inflammatory effects [44] while adopting Mediterranean dietary pattern with levels of fruit, vegetables and whole-grains could reduce inflammation process [45]. Results of current study also showed that choosing more inflammatory diet which characterized by high DII score had a close relationship with increased risk of PCOS disease. This association was remained significant after adjusting for several confounding factors including energy intake, physical activity, BMI and family history of PCOS which shows the profound effects of diet on the incidence of inflammation- related diseases.

As we know, this is the first case-control study for assessing the association between DII and PCOS in the world. Another strengths of this study were including 36 out of 45 dietary parameters for measuring DII score the use of a validated FFQ [27] for obtaining dietary intakes of the participants.

\section{Conclusion}

In conclusion, the results of present study showed that consuming more pro-inflammatory diets with higher DIl scores are associated with increased Odds Ratio (OR) of PCOS incidence compared to diets with low DII scores and the results were remained significant after adjusting for confounding factors including $\mathrm{BMI}$ and physical activity level which can indicate the use of more dietary related anti-inflammatory nutrients and restriction of foods with pro-inflammatory nutrients for prevention and control of PCOS patients.

\section{Limitations}

It should be mentioned that the FFQ tool which was used for collecting dietary information in this study has a recall bias and its results are dependent to the memory of the respondents which was an important limitation of this study. Another limitation of this study was the number of participants which was relatively low for this type of studies.

\section{Abbreviations}

DII: Dietary Inflammatory Index; PCOS: Poly Cystic Ovary Syndrome; CRP: C-Reactive Protein; FFQ: Food Frequency Questionnaire; IL: Interleukin; TNFa: Tumour necrosis factor a; MUFA: Monounsaturated Fatty Acid; PUFA: Polyunsaturated Fatty Acid; SFA: Saturated Fatty Acid

\section{Declarations}

\section{Acknowledgements}

The authors are very thankful to the all patient and control group participants. 


\section{Author contributions}

SS: designing the study and Supervision; PK: sampling and data collection; MB, SS and EYR: Data analysis and/or interpretation; $\mathrm{PK}, \mathrm{EY}$ and SS: drafting the manuscript; $\mathrm{EF}$ and $\mathrm{MM}$ : revising the article for important intellectual content. All authors have approved the final manuscript.

\section{Funding}

This work had no source of funding.

\section{Availability of data and materials}

Data will be obtained up on a reasonable request by emailing to the corresponding author of this paper using "saburi_somaye@yahoo.com".

\section{Declarations Ethics approval and consent to participate}

This study was supported by the School of Health Nutritional of Lorestan University of Medical Sciences (ID: IR.LUMS.REC.1398.058). All participants signed a written informed consent before beginning the study.

\section{Consent for publication}

Not applicable.

\section{Competing interests}

The authors declare that there is no conflict of interest with respect to this manuscript.

\section{References}

1. Beydoun HA, Stadtmauer L, Beydoun MA, Russell H, Zhao Y, Oehninger S: Polycystic ovary syndrome, body mass index and outcomes of assisted reproductive technologies. Reproductive biomedicine online $2009,18(6): 856-863$.

2. Lujan ME, Chizen DR, Pierson RA: Diagnostic criteria for polycystic ovary syndrome: pitfalls and controversies. Journal of obstetrics and gynaecology Canada 2008, 30(8):671-679.

3. Moran C, Arriaga M, Rodriguez G, Moran S: Obesity differentially affects phenotypes of polycystic ovary syndrome. International Journal of Endocrinology 2012, 2012.

4. Merkin SS, Azziz R, Seeman T, Calderon-Margalit R, Daviglus M, Kiefe C, Matthews K, Sternfeld B, Siscovick D: Socioeconomic status and polycystic ovary syndrome. Journal of women's health 2011, 20(3):413-419.

5. Fingert SB, Shah B, Kessler M, Pawelczak M, David R: Evaluation of adolescents for polycystic ovary syndrome in an urban population. Journal of clinical research in pediatric endocrinology 2009, 1(4):188.

6. Meier RK: Polycystic ovary syndrome. Nursing Clinics 2018, 53(3):407-420. 
7. Tehrani FR, Rashidi H, Azizi F: The prevalence of idiopathic hirsutism and polycystic ovary syndrome in the Tehran Lipid and Glucose Study. Reproductive Biology and Endocrinology 2011, 9(1):144.

8. Sayehmiri F, Kiani F, Maleki F, Ahmadi M, Shohani M: Prevalence of polycystic ovary syndrome in Iranian women: a systematic review and meta-analysis. The Iranian Journal of Obstetrics, Gynecology and infertility 2014, 17(115):11-21.

9. Goodarzi MO, Jones MR, Li X, Chua AK, Garcia OA, Chen Y-DI, Krauss RM, Rotter JI, Ankener W, Legro RS: Replication of association of DENND1A and THADA variants with polycystic ovary syndrome in European cohorts. Journal of medical genetics 2012, 49(2):90-95.

10. Wang ET, Calderon-Margalit R, Cedars MI, Daviglus ML, Merkin SS, Schreiner PJ, Sternfeld B, Wellons M, Schwartz SM, Lewis CE: Polycystic ovary syndrome and risk for long-term diabetes and dyslipidemia. Obstetrics and gynecology 2011, 117(1):6.

11. Xiang S-K, Hua F, Tang Y, Jiang X-H, Zhuang Q, Qian F-J: Relationship between serum lipoprotein ratios and insulin resistance in polycystic ovary syndrome. International journal of Endocrinology 2012, 2012.

12. Pastore LM, Patrie JT, Morris WL, Dalal P, Bray MJ: Depression symptoms and body dissatisfaction association among polycystic ovary syndrome women. Journal of psychosomatic research 2011, 71(4):270-276.

13. Gluszak O, Stopinska-Gluszak U, Glinicki P, Kapuscinska R, Snochowska H, Zgliczynski W, Debski R: Phenotype and metabolic disorders in polycystic ovary syndrome. ISRN endocrinology 2012, 2012.

14. Bu Z, Kuok K, Meng J, Wang R, Xu B, Zhang H: The relationship between polycystic ovary syndrome, glucose tolerance status and serum preptin level. Reproductive biology and endocrinology 2012 , 10(1):10.

15. Sidhwani S, Scoccia B, Sunghay S, Stephens-Archer CN, Mazzone T, Sam S: Polycystic ovary syndrome is associated with atherogenic changes in lipoprotein particle number and size independent of body weight. Clinical endocrinology 2011, 75(1):76-82.

16. Moran LJ, Hutchison SK, Norman RJ, Teede HJ: Lifestyle changes in women with polycystic ovary syndrome. Cochrane Database of Systematic Reviews 2011(7).

17. González F, Rote NS, Minium J, Kirwan JP: Increased activation of nuclear factor KB triggers inflammation and insulin resistance in polycystic ovary syndrome. The Journal of Clinical Endocrinology \& Metabolism 2006, 91(4):1508-1512.

18. González F: Inflammation in polycystic ovary syndrome: underpinning of insulin resistance and ovarian dysfunction. Steroids 2012, 77(4):300-305.

19. Peral Bn, San Millán JL, Castello R, Moghetti P, Escobar-Morreale HcF: The methionine 196 arginine polymorphism in exon 6 of the TNF receptor 2 gene (TNFRSF1B) is associated with the polycystic ovary syndrome and hyperandrogenism. The Journal of Clinical Endocrinology \& Metabolism 2002, 87(8):3977-3983.

20. Shivappa N, Steck SE, Hurley TG, Hussey JR, Hébert JR: Designing and developing a literature-derived, population-based dietary inflammatory index. Public health nutrition 2014, 17(8):1689-1696.

21. Neufcourt L, Assmann K, Fezeu L, Touvier M, Graffouillère L, Shivappa N, Hébert J, Wirth M, Hercberg S, Galan P: Prospective association between the dietary inflammatory index and metabolic syndrome: 
Findings from the SU. VI. MAX study. Nutrition, Metabolism and Cardiovascular Diseases 2015, 25(11):988-996.

22. Ruiz-Canela M, Zazpe I, Shivappa N, Hébert JR, Sánchez-Tainta A, Corella D, Salas-Salvadó J, Fito M, Lamuela-Raventós RM, Rekondo J: Dietary inflammatory index and anthropometric measures of obesity in a population sample at high cardiovascular risk from the PREDIMED (PREvencion con Dleta MEDiterranea) trial. British journal of nutrition 2015, 113(6):984-995.

23. Shivappa N, Steck SE, Hurley TG, Hussey JR, Ma Y, Ockene IS, Tabung F, Hébert JR: A population-based dietary inflammatory index predicts levels of C-reactive protein in the Seasonal Variation of Blood Cholesterol Study (SEASONS). Public health nutrition 2014, 17(8):1825-1833.

24. Neufcourt L, Assmann KE, Fezeu LK, Touvier M, Graffouillere L, Shivappa N, Hébert JR, Wirth MD, Hercberg S, Galan P: Prospective association between the dietary inflammatory index and cardiovascular diseases in the SUpplémentation en VItamines et Minéraux AntioXydants (SU. VI. MAX) cohort. Journal of the American Heart Association 2016, 5(3):e002735.

25. Shivappa N, Bosetti C, Zucchetto A, Montella M, Serraino D, La Vecchia C, Hébert JR: Association between dietary inflammatory index and prostate cancer among Italian men. British Journal of Nutrition 2015, 113(2):278-283.

26. Tabung FK, Steck SE, Zhang J, Ma Y, Liese AD, Agalliu I, Hingle M, Hou L, Hurley TG, Jiao L: Construct validation of the dietary inflammatory index among postmenopausal women. Annals of epidemiology 2015, 25(6):398-405.

27. Mirmiran P, Esfahani FH, Mehrabi Y, Hedayati M, Azizi F: Reliability and relative validity of an FFQ for nutrients in the Tehran lipid and glucose study. Public health nutrition 2010, 13(5):654-662.

28. Craig CL, Marshall AL, Sjöström M, Bauman AE, Booth ML, Ainsworth BE, Pratt M, Ekelund U, Yngve A, Sallis JF: International physical activity questionnaire: 12-country reliability and validity. Medicine \& science in sports \& exercise 2003, 35(8):1381-1395.

29. Vasheghani-Farahani A, Tahmasbi M, Asheri H, Ashraf H, Nedjat S, Kordi R: The Persian, last 7-day, long form of the International Physical Activity Questionnaire: translation and validation study. Asian journal of sports medicine 2011, 2(2):106.

30. Moghaddam MB, Aghdam FB, Jafarabadi MA, Allahverdipour H, Nikookheslat SD, Safarpour S: The Iranian Version of International Physical Activity Questionnaire (IPAQ) in Iran: content and construct validity, factor structure, internal consistency and stability. World applied sciences journal 2012, 18(8):1073-1080.

31. Committee IR: Guidelines for data processing and analysis of the International Physical Activity Questionnaire (IPAQ)-short and long forms. http://www ipaq ki se/scoring pdf 2005.

32. Kim H-Y, Lee J, Kim J: Association between dietary inflammatory index and metabolic syndrome in the general Korean population. Nutrients 2018, 10(5):648.

33. Denova-Gutiérrez E, Muñoz-Aguirre P, Shivappa N, Hébert JR, Tolentino-Mayo L, Batis C, Barquera S: Dietary inflammatory index and type 2 diabetes mellitus in adults: the diabetes mellitus survey of Mexico City. Nutrients 2018, 10(4):385. 
34. Vatandoost A, Azadbakht L, Morvaridi M, Kabir A, Farsani GM: Association between dietary inflammatory index and risk of cardiovascular diseases among firefighters. International Journal of Preventive Medicine 2020, 11.

35. Shoelson SE, Lee J, Goldfine AB: Inflammation and insulin resistance. The Journal of clinical investigation 2006, 116(7):1793-1801.

36. Vázquez-Carballo A, Ceperuelo-Mallafré V, Chacón MR, Maymó-Masip E, Lorenzo M, Porras A, Vendrell J, Fernández-Veledo S: TWEAK prevents TNF-a-induced insulin resistance through PP2A activation in human adipocytes. American Journal of Physiology-Endocrinology and Metabolism 2013, 305(1):E101E112.

37. Resnick HE, Jones K, Ruotolo G, Jain AK, Henderson J, Lu W, Howard BV: Insulin resistance, the metabolic syndrome, and risk of incident cardiovascular disease in nondiabetic American Indians: the Strong Heart Study. Diabetes care 2003, 26(3):861-867.

38. Saboori S, Djalali M, Rad EY, Nematipour E, Saboor-Yaraghi AA, Javanbakht MH, Eshraghian MR, Ramezani A, Koohdani F: Various effects of omega 3 and omega 3 plus vitamin E supplementations on serum glucose level and insulin resistance in patients with coronary artery disease. Iranian journal of public health 2016, 45(11):1465.

39. Cowey S, Hardy RW: The metabolic syndrome: a high-risk state for cancer? The American journal of pathology 2006, 169(5):1505-1522.

40. Saboori S, Rad EY, Birjandi M, Mohiti S, Falahi E: Serum insulin level, HOMA-IR and prostate cancer risk: A systematic review and meta-analysis. Diabetes \& Metabolic Syndrome: Clinical Research \& Reviews 2019, 13(1):110-115.

41. Repaci A, Gambineri A, Pasquali R: The role of low-grade inflammation in the polycystic ovary syndrome. Molecular and cellular endocrinology 2011, 335(1):30-41.

42. Kaya C, Pabuçcu R, Koca C, Oğuz AK, Erkan AF, Korkmaz A, Erbaş D: Relationship between interleukin-6 levels and ambulatory blood pressure in women with polycystic ovary syndrome. Fertility and sterility 2010, 94(4):1437-1443.

43. Goodarzi MO, Korenman SG: The importance of insulin resistance in polycystic ovary syndrome. Fertility and sterility 2003, 80(2):255-258.

44. Barbaresko J, Koch M, Schulze MB, Nöthlings U: Dietary pattern analysis and biomarkers of low-grade inflammation: a systematic literature review. Nutrition reviews 2013, 71(8):511-527.

45. Schwingshackl L, Hoffmann G: Mediterranean dietary pattern, inflammation and endothelial function: a systematic review and meta-analysis of intervention trials. Nutrition, Metabolism and Cardiovascular Diseases 2014, 24(9):929-939. 